УДК 621.774 .3

В. Ф. Балакин /д. т. н./

Национальная металлургическая академия

Украины, г. Днипро, Украина

В. Д. Добряк /к. т. н./, Ю. Д. Угрюмов /к. т. н./

ГП «Укргипромез», г. Днипро, Украина

\title{
Исследование отката гильзы в процессе пилигримовой прокатки
}

\author{
V. F. Balakin /Dr. Sci. (Tech.)/ \\ V. D. Dobryak /Cand. Sci. (Tech.)/, \\ Yu. D. Ugryumov /Cand. Sci. (Tech.)/
}

National Metallurgical Academy of Ukraine, Dnipro, Ukraine

SE "Ukrgipromez", Dnipro, Ukraine

\section{Study of roll backing in the process pilgrim rolling}

Цель. Исследование основных фракторов, влияющих на откат гильзы валками пилигримового стана, что, в конечном счете, оказывает влияние на синхронизацию работы системы «валки - подающий аппарат» и снижение производительности пилигримового стана, а также нестабильную, с ударами в валки гильзы работу подающего аппарата и главной линии стана.

Методика. Использование метода баланса работ всех сил, в том числе силы инерции, действующих на подвижные части подающего аппарата вместе с гильзой и дорном после выхода гильзы из контакта с валками.

Результаты. Приведены результаты теоретических и экспериментальных исследований влияния основных фракторов на откат гильзы. С помощью использованной методики дана количественная и качественная оценка влияния исследованных фракторов на процесс торможения и косвенного влияния их на степень синхронизации работы подающего аппарата и валков.

Научная новизна. Установлены основные фракторы, влияющие на величину отката подвижных частей подающего аппарата вместе с гильзой и дорном в процессе пилигримовой прокатки, в том числе на величину отката по инерции. Величина пути отката по инерции для конкретных условий при коэффрициенте трения скольжения равном 0,2 составила 45 мм, что согласуется с данными, полученными на пильгерстане 5-12" с помощью видеосъемки. Исследованиями установлено, что при увеличении давления в воздушной камере на 20 \% по отношению к заданному путь торможения по инерции уменьшается на 16,6 \%, а при уменьшении этого давления на $20 \%$, путь торможения увеличивается на $21,8 \%$.

Практическая значимость. Результаты проведенных теоретических и экспериментальных исследований могут быть использованы при разработке автоматической системы синхронизации «валки - подающий аппарат», что позволит повысить производительность за счет стабилизации подачи гильзы в валки в каждом цикле прокатки, а также стабильность и надежность работы пилигримового стана без ударов гильзы в валки. (Ил. 3. Табл. 2. Библиогр.: 6 назв.)

Ключевые слова: труба, гильза, пилигримовый стан, дорн, подающий аппарат, синхронизация, подача, производительность, скорость прокатки, откат.

DOI: $10.33101 / \mathrm{s} 005-2035580$

Введение. Особенностью процесса горячей пилигримовой прокатки труб, предложенного в 1891 г. братьями Маннесманн, является прокатка гильзы на дорне в трубу в валках, имеющих переменный профиль в продольном и поперечном сечениях (рис. 1).

Рабочий валок пилигримового стана (в продольном сечении по вершине калибра) может быть разбит на два основных участка: рабочий $\theta_{p}$ и холостой $\theta_{x x}$. В свою очередь, рабочий участок состоит из бойка $\theta_{\delta}$ полирующего участка $\theta_{n}$ и продольного выпуска $\theta_{n 6}$. В бочку валка врезан

калибр, обеспечивающий получение наружного профиля трубы (поперечное сечение валка).

Наличие рабочего и холостого участков валка связано с тем, что пилигримовый процесс двухтактный. При этом каждый цикл (такт) состоит из рабочего и холостого ходов. В процессе рабочего хода валков происходит деформация гильзы участками бойка $\theta_{\delta}, \theta_{n}$ и $\theta_{n b}$ в направлении вращения валков или так называемый откат гильзы валками.

При откате в подающем аппарате образуется воздушная «пружина». Откат продолжается до 

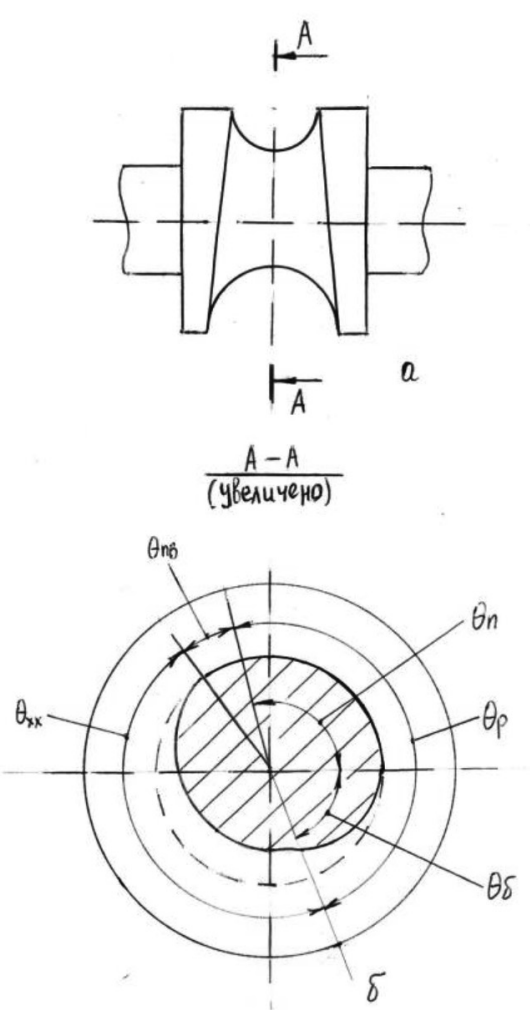

Рис. 1. Рабочий валок пилигримового стана: а - общий вид, б - сечение А-А

тех пор, пока подпор со стороны подающего аппарата не нарушит контакт металла с валками, перемещаясь еще определенное время по инерции, движущиеся массы под воздействием сжатого воздуха через некоторое время останавливаются.

Таким образом, величина отката в процессе рабочего хода состоит из:

$$
L_{0}=\ell_{\delta}+\ell_{n}+\ell_{n b}+\ell_{u}
$$

где $L_{0}$ - величина отката в процессе рабочего хода; $\ell_{\delta}$ - величина отката участком бойка; $\ell_{n}$ - величина отката полирующим участком; $\ell_{n b}-$ величина отката продольным выпуском; $\ell_{u}$ - величина отката по инерции.

Затем наступает холостой ход (или накат), когда движущиеся массы после остановки изменяют направление движения на обратное (навстречу к валкам) и возвращаются в исходное положение для очередного цикла деформации.

Величина наката состоит из пути разгона подвижных частей за счет давления воздуха в воздушной камере подающего аппарата и пути их торможения для остановки в исходном положении.

При этом холостой участок валка имеет в каждый рассматриваемый момент времени такой калибр, чтобы обеспечить свободное прохождение гильзы при накате, а протяженность холостого участка валка должна обеспечить возвращение движущихся масс в исходное положение. Последнее условие лежит в основе синхронизации работы системы «валки - подающий аппарат».
В работе [1] приведены различные варианты представления условия синхронизации, одно из которых имеет вид:

$$
\tau_{h}+\tau_{b} \gtrless 30\left(\theta_{x x}-K \theta_{n b}\right) / \pi n b
$$

где $\tau_{h}$ - время отката по инерции, с; $\tau_{b}$ - время возвращения подвижных частей в исходное положение (время наката), с; $\theta_{x x}$ - центральный угол холостого участка валка, рад; $\theta_{n \beta}$ - центральный угол продольного выпуска валка, рад; К - коэффициент использования угла продольного выпуска при откате; $n b$ - число оборотов валков, об/мин.

Поскольку работа подающего аппарата и калибровка валков - величины друг от друга независящие, между ними может быть любой знак неравенства в выражении (2).

Наименее исследованным является период отката подвижных частей по инерции.

Целью работы является теоретическое и экспериментальное исследование влияния параметров пилигримовой прокатки труб на величину отката гильзы и подвижных частей подающего аппарата по инерции.

Изложение основных результатов исследования. В период наката отсутствует деформация гильзы валками, поэтому определение скоростей, ускорений и перемещений подвижных частей с гильзой относится к области механики, и к настоящему времени эта задача решена аналитически с достаточной степенью точности [2; 3].

В период собственно прокатки гильзы (период отката) происходит пластическая деформация гильзы валками, при этом валки сообщают подвижным частям с гильзой кинетическую энергию, преодолевая сопротивление воздушного цилиндра подающего аппарата и трения. Для определения скоростей гильзы в этот период введено понятие катающего радиуса валков, который определяют экспериментальным или расчетным путем. Установлено [4], что катающий радиус $R_{\kappa}$ может быть выражен через радиус $R_{n}$ основного профиля пильгервалка в зоне полировки: $R_{\kappa}=\xi \cdot R_{n}$ (табл. 1$)$.

\section{Коэффициенты для определения катающего радиуса валка}

\begin{tabular}{|c|c|c|}
\hline $\begin{array}{c}\text { Зона профиля } \\
\text { пильгервалка }\end{array}$ & Коэффициент, $\xi$ & $\begin{array}{c}\text { Среднее } \\
\text { значение, } \xi_{c p}\end{array}$ \\
\hline $\begin{array}{c}\text { Поек } \\
\text { Полирующий } \\
\text { участок }\end{array}$ & $1,1 \div 1,2$ & 1,15 \\
$1,04 \div 1,08$ & 1,06 \\
\hline
\end{tabular}

Следовательно, воображаемая длина дуги катающего радиуса, ограниченная центральным углом соответствующей зоны основного профиля валка, равна части полного пути отката гильзы. 
Сумма длин дуг, катающих радиусов рабочей части профиля валка будет минимальной оценкой пути отката гильзы и подвижных частей подающего аппарата. Полный путь отката включает также путь торможения по инерции после того, как гильза выйдет из силового контакта с калибром валков. Как показали наши исследования на промышленном пилигримовом стане 5-12", разброс путей отката подвижных частей с гильзой достигает $\pm 15 \%$ от среднего отката.

Разброс величины пути отката гильзы вызывает разброс пути наката. Этот разброс приводит к тому, что в каждом новом цикле прокатки изменяются условия захвата гильзы валками - от принудительного захвата до затравочного, соответственно, не выполняются условия синхронизации работы валков и подающего аппарата. Поэтому важно выяснить, какие факторы оказывают наибольшее влияние на разброс пути торможения подвижных частей подающего аппарата с гильзой после выхода гильзы из силового контакта с валками.

Торможение подвижных частей, куда входят гильза с дорном, дорновой замок и дорновое кольцо, соединительный ниппель и шток с поршнем, в конце отката осуществляется за счет сжатия воздуха в воздушном цилиндре подающего аппарата и сил трения, возникающих в местах контакта подвижных частей с опорными поверхностями (рис. 2).

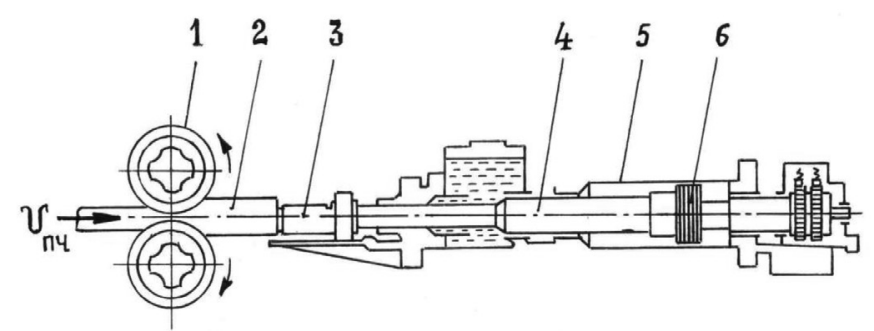

Рис. 2. Схема отката подвижных частей подающего аппарата пилигримового стана:

1 - валки пилигримового стана; 2 - гильза;

3 - дорновой замок; 4 - шток; 5 - воздушный цилиндр; 6 - поршень

Напишем баланс работ всех сил, действующих на подвижные части в период торможения после выхода гильзы из силового контакта с валками:

$$
A_{u н}=A_{c ж}+A_{m p}
$$

где $A_{\text {ин }}$ - работа силы инерции подвижных частей до их полной остановки, равная кинетической энергии этих частей, полученной в результате прокатки.

$$
A_{u н}=\frac{m_{n u} \cdot v_{n u}^{2}}{2},
$$

где $m_{n u}$ - масса подвижных частей; $v_{n u}$ - скорость подвижных частей в момент разрыва силового контакта гильзы с валками пилигримового стана. $A_{c ж}=-\int_{V_{0}}^{V} \rho d V-$ работа сжатия воздуха в воздушном цилиндре подающего аппарата. Как известно [5], работа сжатия воздуха определяется по выражению:

$$
A_{c \kappa}=-\frac{\rho_{0} V_{0}-\rho V}{\kappa-1}=-\frac{p_{0}\left(V_{0}-\frac{p}{p_{0}} V\right)}{\kappa-1}
$$

где $\rho_{0}$ - давление в воздушном цилиндре в момент начала торможения подвижных частей; $V_{0}$ - объем воздуха под поршнем в тот же момент; $p$ - давление воздуха под поршнем в момент остановки подвижных частей; $V$ - объем воздуха под поршнем в момент остановки; $K=1,4$ - показатель адиабаты (при отсутствии теплообмена).

Представим начальный объем в виде двух слагаемых:

$$
V_{0}=V+F_{n} \cdot \ell_{u}
$$

где $F_{n}$ - площадь поршня активная; $\ell_{u}$ - путь торможения.

С другой стороны, тот же объем представим в виде объема условного, так называемого приведенного цилиндра с высотой $h_{0}$ :

$$
V_{0}=h_{0} \cdot E_{n} .
$$

Подставляя (7) в (6), получаем:

$$
V=E_{n} \cdot\left(h_{0}-\ell_{u}\right)
$$

Можно показать, что в адиабатическом процессе сжатия воздуха в воздушном цилиндре справедливо соотношение:

$$
\frac{p}{p_{0}}=\left(1-\frac{\ell_{u}}{h_{0}}\right)^{-k} .
$$

Подставляя (9) и (8) в (5), получаем:

$$
A_{c \varkappa}=-\frac{p_{0}}{k-1}\left[V_{0}-F_{n} \cdot\left(h_{0}-\ell_{u}\right) \cdot\left(1-\frac{\ell_{u}}{h_{0}}\right)^{-k}\right] .
$$

Работу сил трения запишем в виде выражения:

$$
A_{m p}=G_{n u} \cdot f \cdot \ell_{u}
$$

где $G_{n u}$ - вес подвижных частей; $f$ - коэффициент трения скольжения.

С учетом выражений (4), (10), (11) уравнение (3) баланса работ выглядит следующим образом:

$$
\begin{aligned}
& m_{n u} \cdot v_{n u}^{2}=-\frac{2 p_{0}}{\kappa-1}\left[V_{0}-F_{n} \cdot\left(h_{0}-\ell_{u}\right) \cdot\left(1-\frac{\ell_{u}}{h_{0}}\right)^{-k}\right]+ \\
& +2 G_{n u} \cdot f \cdot \ell_{u} .
\end{aligned}
$$

Выразить из этого уравнения в явном виде путь торможения $\ell_{\text {и }}$ не представляется возможным, поэтому перейдем к численному решению и оценим влияние каждого параметра на путь торможения.

В качестве примера принимаем следующие реальные параметры пилигримовой прокатки труб: 
- прокатываемая труба $\mathrm{D}_{\mathrm{T}} \times \mathrm{S}_{\mathrm{T}}=324 \times 9 \mathrm{Mм}$;

- наружный диаметр гильзы D2 = 460 мм;

- масса подвижных частей подающего аппарата $m_{n u}=11208 \mathrm{Hc}^{2} / \mathrm{M}$;

- вес подвижных частей $G_{n u}=109950,5$ H;

- число оборотов валков пилигримового стана $n=60$ об/мин;

- радиус полирующего участка валка $R_{n}=$ $=263$ мM;

- катающий (по вершине калибра) радиус в зоне полирующего участка $R_{\kappa}=1,06 \cdot 263=279$ мм;

- скорость гильзы (подвижных частей) при выходе из полирующего участка

$$
\mathrm{v}_{n u}=\frac{\pi n}{30} \cdot R_{\kappa}=\frac{3,14 \cdot 60}{30} \cdot 0,279=1,75 \mathrm{M} / \mathrm{c} ;
$$

- объем воздуха под поршнем воздушного цилиндра в момент начала торможения $V_{0}=0,48 \mathrm{~m}^{3}$;

- давление воздуха под поршнем в момент начала торможения $p_{0}=83 \cdot 10^{4} \mathrm{H} / \mathrm{m}^{2}$;

- активная площадь поршня $F_{n}=0,44 \mathrm{~m}^{2}$;

- приведенная длина воздушного цилиндра $\mathrm{h}_{0}=\frac{V_{0}}{F_{n}}=\frac{0,48}{0,44}=1,09 \mathrm{M}$.

После подстановки численных значений параметров в уравнение (12) имеем:

$$
\begin{gathered}
34324,5-219901 \cdot f \\
\ell_{u}=-415 \cdot 10^{4}\left[0,48-0,44\left(1,09-\ell_{u}\right) \cdot\left(1-\frac{\ell_{u}}{1,09}\right)^{-1,4}\right] .
\end{gathered}
$$

Придавая различные значения коэффициенту трения, в результате решения уравнения (13) получаем соответствующие пути торможения (табл. 2). Из табл. 2 видно, что при $f=0,2$ путь торможения на $\frac{0,048-0,045}{0,048} \cdot 100=6,2 \%$ меньше, чем при отсутствии трения $(f=0)$, а при $f=0,3$ на $\frac{0,048-0,044}{0,048} \cdot 100=8,33 \%$ меньше, чем при $f=0$. Следовательно, можно не учитывать влияние трения скольжения подвижных частей на путь торможения в пределах $f=0 \div 0,3$. При этом погрешность не превысит $10 \%$.

Таблица 2

Изменение пути торможения подвижных частей в зависимости от коэффициента трения $f$

\begin{tabular}{|c|c|c|c|}
\hline$f$ & 0 & 0,2 & 0,3 \\
\hline$\ell_{u^{\prime}} \mathrm{M}$ & 0,048 & 0,045 & 0,044 \\
\hline
\end{tabular}

Как показали наши измерения с помощью видеосъемки на промышленном пилигримовом стане 5-12", путь торможения подвижных частей подающего аппарата в конце отката при прокатке трубы $\mathrm{D}_{\mathrm{T}} \times \mathrm{S}_{\mathrm{T}}=324 \times 9$ мм составил в среднем $45 \mathrm{MM}$, что говорит о хорошем совпадении теоретического и реального путей торможения.

Оценку влияния давления $\mathrm{p}_{0}$ воздуха в цилиндре подающего аппарата на путь торможения выполним по уравнению (14), полученному из уравнения (12) при подстановке в него тех же числовых параметров пилигримовой прокатки и при нулевом коэффициенте трения.

$$
-p_{0}\left[0,48-0,44\left(1,09-\ell_{u}\right) \cdot\left(1-\frac{\ell_{u}}{1,09}\right)^{-1,4}\right]
$$

Расчеты показали, что при увеличении $p_{0}$ на $20 \%$ по отношению к заданному $p_{0}=83 \cdot 10^{4} \mathrm{H} / \mathrm{m}^{2}$ путь торможения уменьшается на 16,6 \% по отношению $\ell_{u}=0,048$ м (табл. 2); а при уменьшении $p_{0}$ на $20 \%$ путь торможения увеличивается на 21,8 \%. Следовательно, приближенно можно считать, что давление под поршнем в конце отката подвижных частей подающего аппарата влияет обратно пропорционально на путь торможения. Известно [6], что давление воздуха в поршневой полости воздушного цилиндра подающего аппарата с каждым пильгерным шагом понемногу возрастает. Следовательно, уменьшается путь торможения подвижных частей в конце отката и весь путь отката гильзы; нарушаются условия захвата гильзы валками и синхронизация оборотов валков и периода возвратного движения гильзы. Операторы станов, стравливая воздух из поршневой полости, не только снижают подпор гильзы со стороны подающего аппарата и вероятность проскальзывания валков, но и в некоторой степени стабилизируют величину отката подвижных частей.

Оценку влияния начального объема $\mathrm{V}_{0}$ воздуха согласно формуле (12) выполним по уравнению (15), которое получено из (12) путем подстановки в него числовых параметров пилигримовой прокатки при $f=0$.

$$
0,00827=-V_{0}+\left(V_{0}-0,44 \ell_{u}\left(1-\frac{0,44 \ell_{u}}{V_{0}}\right)^{-1,4} .\right.
$$

Расчеты по уравнению (15) показали, что как при увеличении, так и при уменьшении начального объема воздуха на 20 \% по отношению к заданному $V_{0}=0,48 \mathrm{~m}^{3}$ путь торможения уменьшается приблизительно на $6 \%$ по отношению к $\ell_{u}=$ $=0,048$ м (табл. 2). Это говорит о том, что объем воздушной поршневой полости подающего аппарата не оптимален. Его следовало бы уменьшить, что способствовало бы не только уменьшению пути торможения подвижных частей в конце отката, но и повысило бы степень сжатия воздуха.

Оценку влияния скорости $\mathbf{v}_{n и}$ подвижных частей подающего аппарата согласно формуле (12) выполним по уравнению (16), которое получено из (12) путем подстановки в него числовых параметров при $f=0$.

$$
\mathrm{v}_{n u}-19,24 \sqrt{0,44\left(1,09-\ell_{u}\left(1-\frac{\ell_{u}}{1,09}\right)^{-1,4}\right.} .
$$


Уравнение (16) представлено в виде графика на рис. 3. Оно аппроксимируется линейной функцией (17) с погрешностью $1,5 \div 2 \%$ :

$$
\ell_{u}=0,051 \mathrm{v}_{n u}-0,041 .
$$

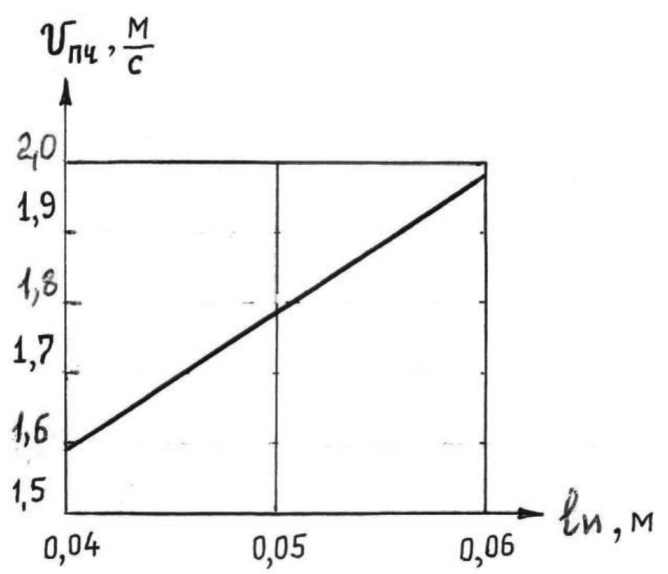

Рис. 3. График зависимости пути торможения подвижных частей подающего аппарата от скорости в момент выхода гильзы из контакта с валками

Из графика (рис. 3) и формулы (17) следует, что увеличение или уменьшение скорости подвижных частей, например на 20 \%, вызывает увеличение или уменьшение пути торможения на $37 \%$, т.е. почти в 2 раза.

Проверка по уравнению (12) влияния массы подвижных частей на путь торможения, как и следовало ожидать, показала приблизительно пропорциональное изменение пути торможения. Это следует иметь в виду в случае изменений массы подвижных частей или только гильзы.

\section{Выводы}

1. Из всех рассмотренных факторов (коэффициент трения подвижных частей, давление воздуха в поршневой полости цилиндра подающего аппарата, объем воздуха в той же полости, масса и скорость подвижных частей подающего аппарата) наибольшее влияние на путь торможения подвижных частей в конце отката оказывает скорость подвижных частей, которую они получают в момент отрыва гильзы от валков пилигримового стана.

2. При изменении скоростного режима прокатки гильзы в сторону увеличения числа оборотов валков увеличивается путь торможения и в целом путь отката подвижных частей, что приводит к уменьшению подачи гильзы в валки, а уменьшение числа оборотов валков уменьшает путь торможения и в целом путь отката подвижных частей, что приводит к увеличению подачи гильзы в валки. И в том, и в другом случае нарушается синхронная работа подающего аппарата и валков пилигримового стана.
Библиографический список/References

1. Экономия металла при производстве труб нефтяного сортамента / А. А. Чернявский, В. В. Березовский, Ю. Д. Угрюмов. - М.: Металлургия, 1987. - 304 с.

Chernyavskiy A. A., Berezovskiy V. V., Ugryumov Yu. D. Ekonomiya metalla pri proizvodstve trub neftyanogo sortamenta. Moscow, Metallurgiya, 1987, $304 \mathrm{p}$.

2. Праздников А. В. Гидропривод в металлургии / А. В. Праздников. - М.: Металлургия, 1973. $336 \mathrm{c}$.

Prazdnikov A. V. Gidroprivod v metallurgii. Mosocow, Metallurgiya, 1973, 336 p.

3. Оборудование цехов с пилигримовыми трубопрокатными установками / С. Н. Кожевников, А. В. Праздников, А. М. Иоффе и др.; под ред. С. Н. Кожевникова. - М.: Металлургия, 1974. $254 \mathrm{c}$.

Kozhevnikov S. N., Prazdnikov A. V., Ioffe A. M. Oborudovanie tsekhov s piligrimovymi truboprokatnymi ustanovkami. Moscow, Metallurgiya, 1974, 254 p.

4. Матвеев Ю. М. Калибровка валков и инструмента трубных станов / Ю. М. Матвеев, Я. Л. Ваткин. - М.: Металлургиздат, 1951. - 412 с.

Matveev Yu. M., Vatkin Ya. L. Kalibrovka valkov $i$ instrumenta trubnykh stanov. Moscow, Metallurgizdat, 1951, $412 \mathrm{p}$.

5. Цыпкин Я. 3. Основы теории автоматических систем / Я. 3. Цыпкин. - М.: Наука, 1977. 560 c.

Tsypkin Ya. Z. Osnovy teorii avtomaticheskikh sistem. Moscow, Nauka, 1977, 560 p.

6. Особенности гидравлического механизма подачи гильзы в валки пилигримового стана и пути его усовершенствования / В. Д. Добряк, В. Ф. Балакин, Ю. Д. Угрюмов и др. // Черная металлургия. - 2013. - № 2. - С. 62-77.

Dobryak V. D., Balakin V. F., Ugryumov Yu. D. Osobennosti gidravlicheskogo mekhanizma podachi gil'zyv valki piligrimovogo stana i puti ego usovershenstvovaniya. Chernaya metallurgiya, 2013, no. 2, pp. 62-77.

Мета. Дослідження впливу основних фракторів, що впливають на відкат гільзи валками пілігримового стану, що в кінцевому рахунку впливає на синхронізацію роботи системи «валки - подавальний апарат» і зниження продуктивності пілігримового стану, а також нестабільну, з ударами в валки гільзи роботу подавального апарату і головної лінії стану.

Методика. Використання методу балансу робіт усіх сил, у тому числі сили інериії, що діють на рухомі частини подавального апарату разом з гільзою і дорном після виходу гільзи з контакту з валками.

Результати. Наведено результати теоретичних та експериментальних досліджень впливу основних факторів на відкат гільзи. За допомогою використаної методики подано кількісну та якісну оцінка впливу досліджених фракторів на процес гальмування і не- 
прямого впливу їх на ступінь синхронізації роботи подавального апарату і валків.

Наукова новизна. Встановлено основні фрактори, що впливають на величину відкату рухомих частин подавального апарату разом з гільзою і дорном у процесі пілігримової прокатки, в тому числі на величину відкату за інерцією. Величина шляху відкату за інерцією для конкретних умов при коефіцієнті тертя ковзання, який дорівнює 0,2, склала 45 мм, що узгоджується з даними, отриманими на пільгерстані 5-12" за допомогою відеозйомки. Дослідженнями встановлено, що при збільшенні тиску в повітряній камері на 20 \% у відношенні до заданого шлях гальмування за інерцією зменшується на 16,6 \%, а при зменшенні цього тиску на 20 \% шлях гальмування збільшується на 21,8\%.

Практична значущість. Результати проведених теоретичних і експериментальних досліджень можуть бути використані при розробці автоматичної системи синхронізації «валки - подавальний апарат», що дозволить підвищити продуктивність за рахунок стабілізації подачі гільзи у валки в кожному циклі прокатки, а також стабільність і надійність роботи пілігримового стану без ударів гільзи у валки.

Ключові слова: труба, гільза, пілігримовий стан, дорн, подавальний апарат, синхронізація, подача, продуктивність, швидкість прокатки, відкат.

Purpose. The study of the influence of the main factors influencing the rollback of the liner with pilger mill rolls, which ultimately affects the synchronization of the "rolls feeder" system and the performance of the pilger mill, as well as the work of the feeder and the main line that are not stable with blows in the liner rolls the camp.

Methodology. The use of the balance method of all forces, including the forces of inertia acting on the moving parts of the feeder, together with the sleeve and the mandrel after the sleeve leaves the contact with the rollers.

Finding. The article presents the results of theoretical and experimental studies of the influence of the main factors on the rollback of the liner. Using the methods used, a quantitative and qualitative assessment of the influence of the factors studied on the braking process and their indirect influence on the degree of synchronization of the feeding apparatus and rolls is given.

Originality. The main factors affecting the amount of recoil of the moving parts of the feeding apparatus along with the sleeve and mandrel in the process of pilgrim rolling, including the amount of recoil by inertia, are established. The size of the rollback path by inertia for specific conditions with a sliding friction coefficient of 0.2 was $45 \mathrm{~mm}$, which is consistent with the data obtained on pilgerstan 5-12" using video filming. Research has shown that when the pressure in the air chamber increases by $20 \%$ relative to to a given point, the inertia deceleration path decreases by $16.6 \%$, and when this pressure decreases by $20 \%$, the deceleration path increases by $21.8 \%$.

Practical value. The results of theoretical and experimental studies can be used in the development of an automatic synchronization system "rolls - feeding device", which will improve performance by stabilizing the feed of the liner to the rolls in each rolling cycle, as well as the stability and reliability of the piligrimage mill without impact of the liner into the rolls.

Key words: pipe, liner, pilger mill, mandrel, feeder, synchronization, feed, capacity, rolling speed, rollback.

Рекомендована к публикации
д. т. н. О. П. Максименко
Поступила 25 ноября 2018

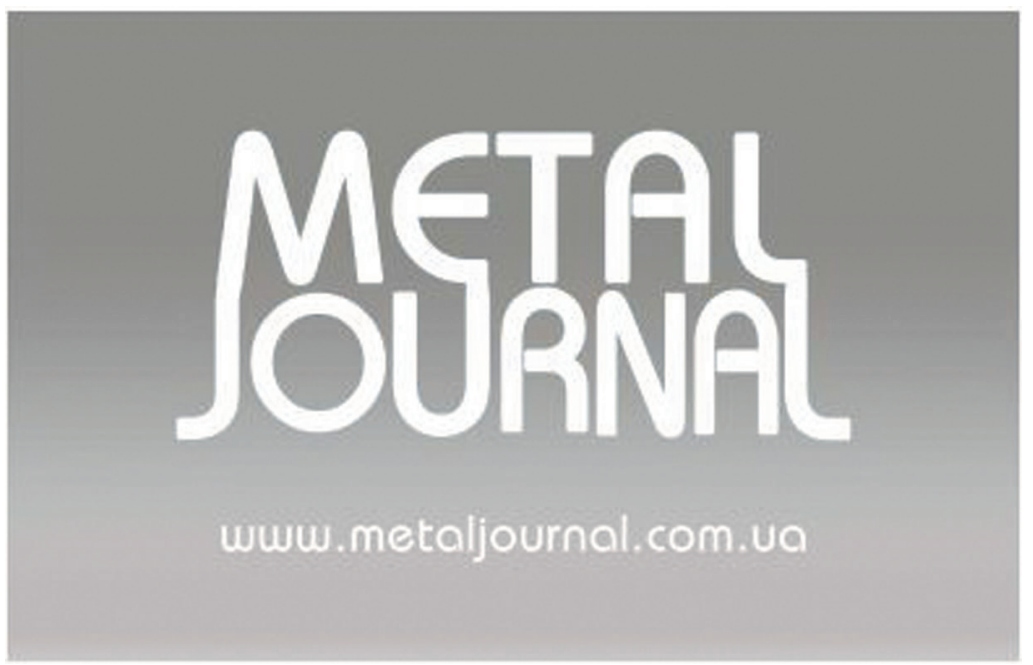

$\overline{\text { (с) Металмургическая и горнорудная промышленность/2018 } 5}$ 